\title{
Publisher Correction: B cells in autoimmune and neurodegenerative central nervous system diseases
}

\author{
Joseph J. Sabatino Jr, Anne-Katrin Pröbstel(D) and Scott S. Zamvil (D)
}

Nature Reviews Neuroscience (2019) https://doi.org/10.1038/s41583-019-0233-2 Published online 11 November 2019

The affiliations for two of the authors were incorrect. The affiliations for Anne-Katrin Pröbstel should have been listed as: Department of Neurology, University of California, San Francisco, San Francisco, CA, USA; Weill Institute for Neurosciences, University of California, San Francisco, San Francisco, CA, USA; and Neurologic Clinic and Policlinic, Departments of Medicine and Biomedicine, University Hospital Basel and University of Basel, Basel, Switzerland. The affiliations for Scott Zamvil should have been listed as: Department of Neurology, University of California, San Francisco, San Francisco, CA, USA; Weill Institute for Neurosciences, University of California, San Francisco, San Francisco, CA, USA; and Program in Immunology, University of California, San Francisco, San Francisco, CA, USA. The affiliations have been updated in the online version of this article.

https://doi.org/10.1038/s41583-019-0251-0 I Published online 26 November 2019 